\title{
Characterization of the Amino Acid Adducts of the Enedial Derivative of Teucrin A
}

Alexandra Druckova and Lawrence J. Marnett*

Departments of Biochemistry, Chemistry, and Pharmacology

Vanderbilt Institute of Chemical Biology

Center in Molecular Toxicology

Vanderbilt-Ingram Cancer Center

Vanderbilt University School of Medicine

Nashville TN 37232-0146

Supporting Information 
Suppl. Figure 1. ${ }^{1} \mathrm{H}$ NMR spectrum of $\mathbf{3 a}(400 \mathrm{MHz})$.

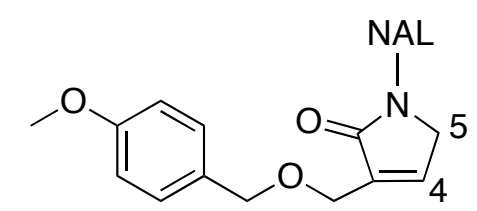

3a major

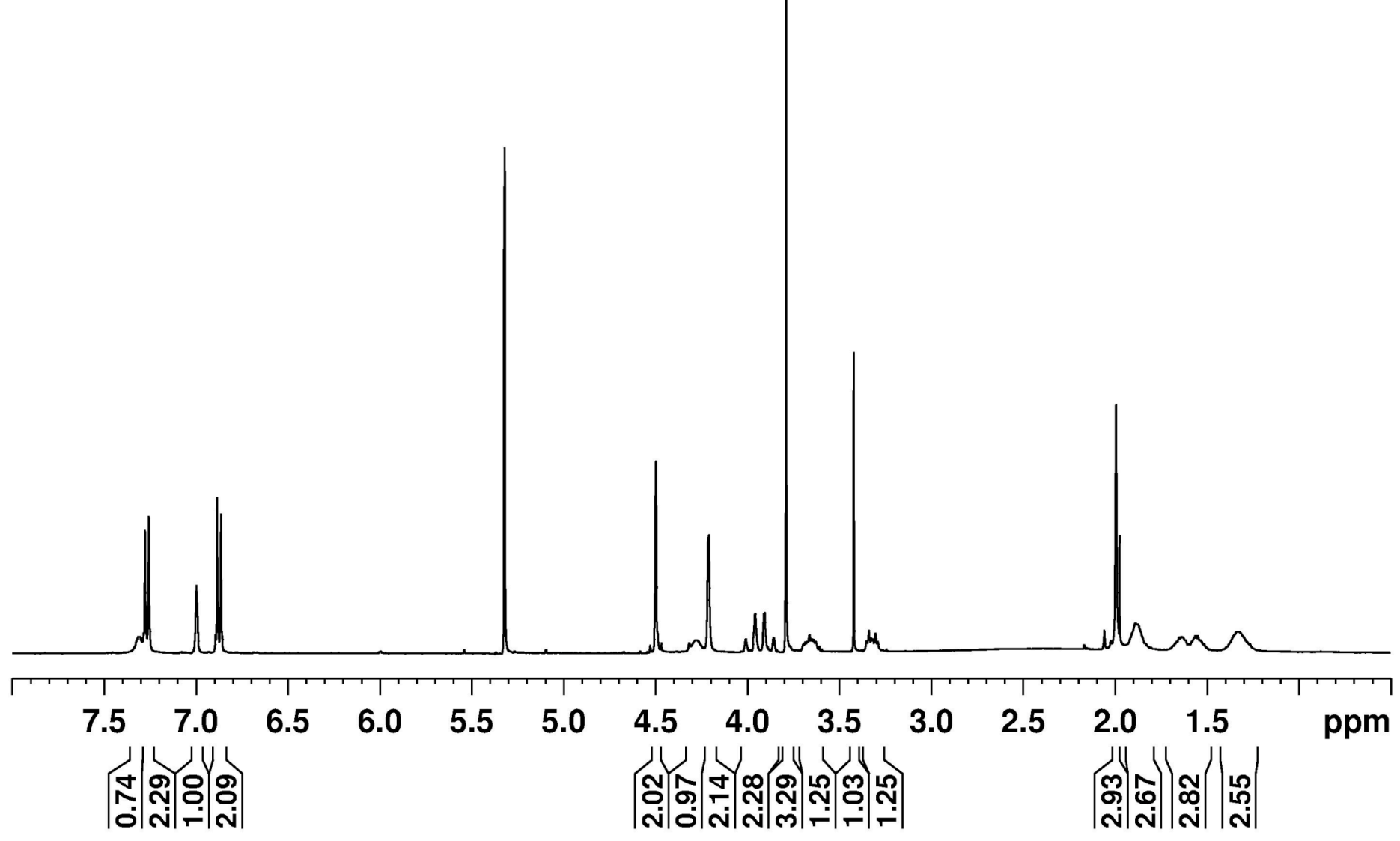


Suppl. Figure 2. COSY spectrum of $\mathbf{3 a}(400 \mathrm{MHz})$.

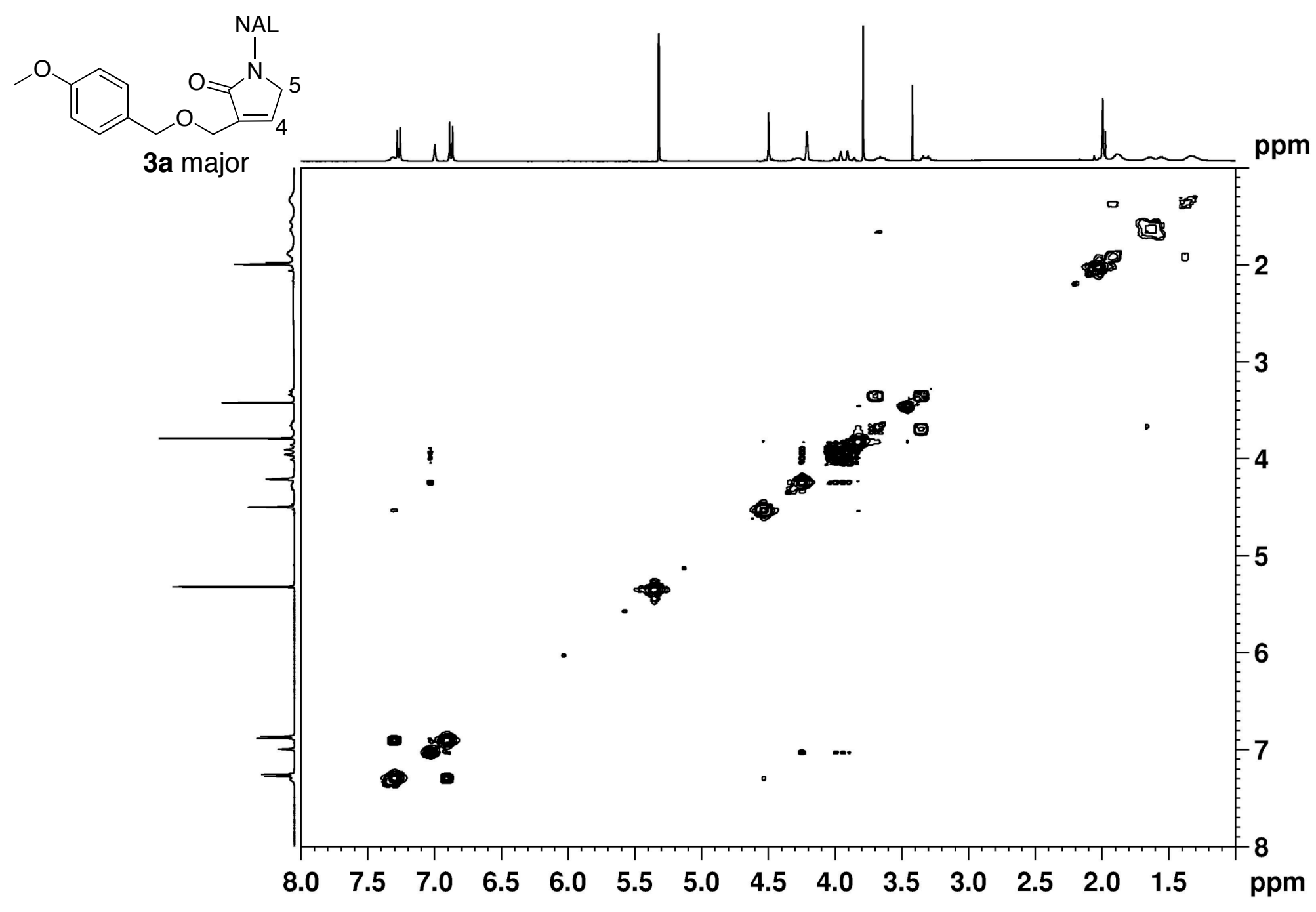


Suppl. Figure 3. ${ }^{1} \mathrm{H}$ NMR spectrum of $3 \mathbf{b}(400 \mathrm{MHz})$.

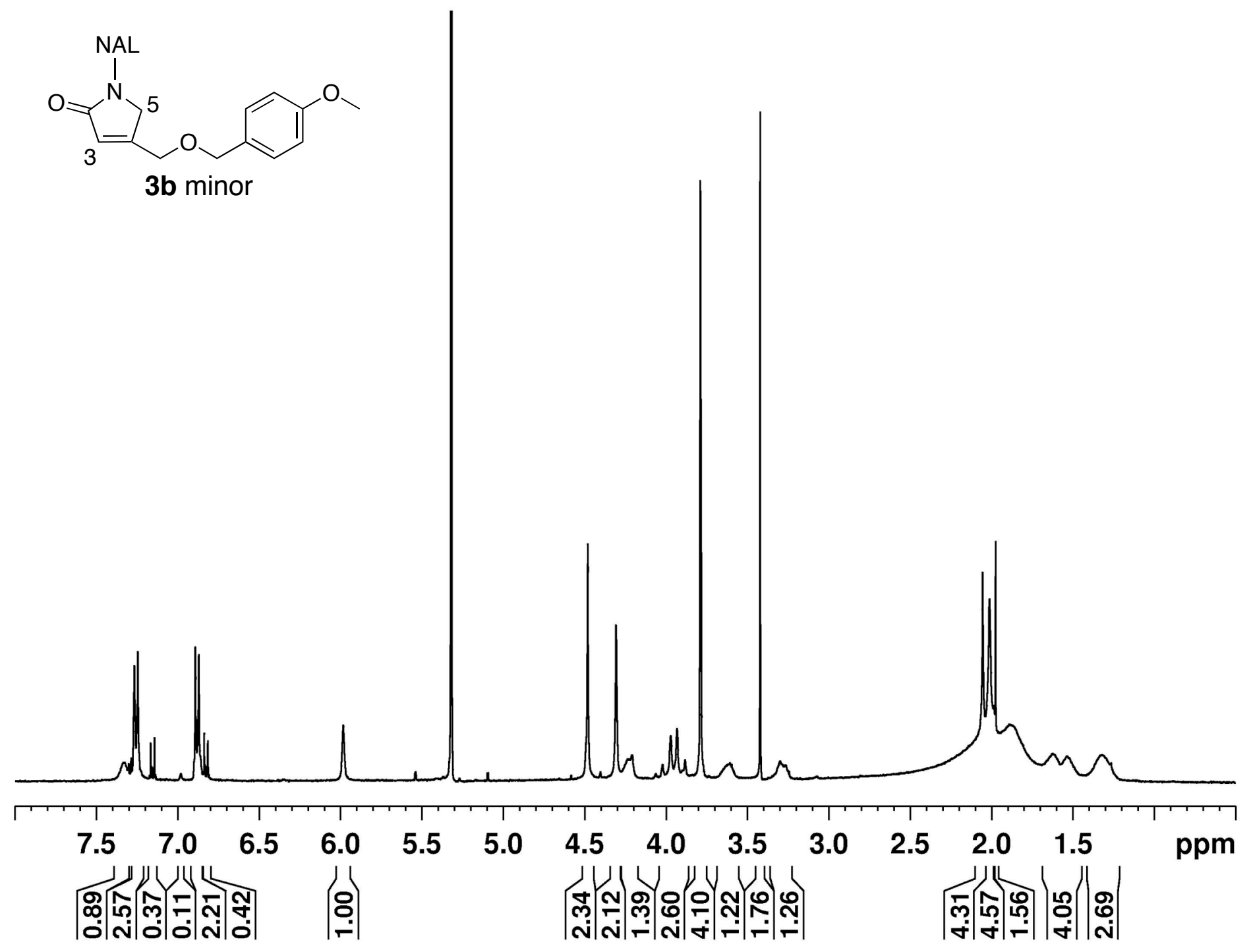


Suppl. Figure 4. COSY spectrum of $\mathbf{3 b}(400 \mathrm{MHz})$.

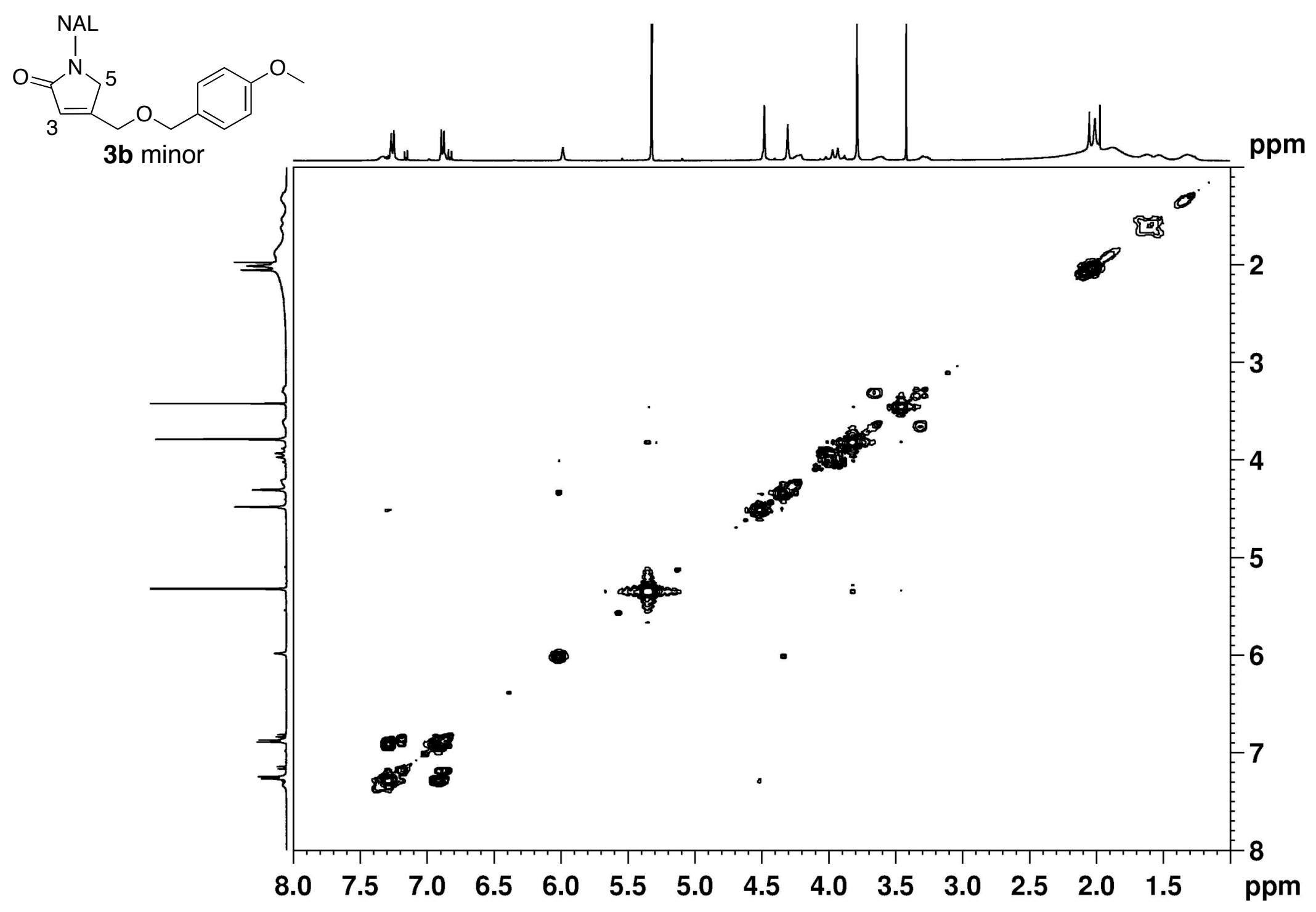


Suppl. Figure 5. ${ }^{1} \mathrm{H}$ NMR spectrum of 4 (400 MHz).
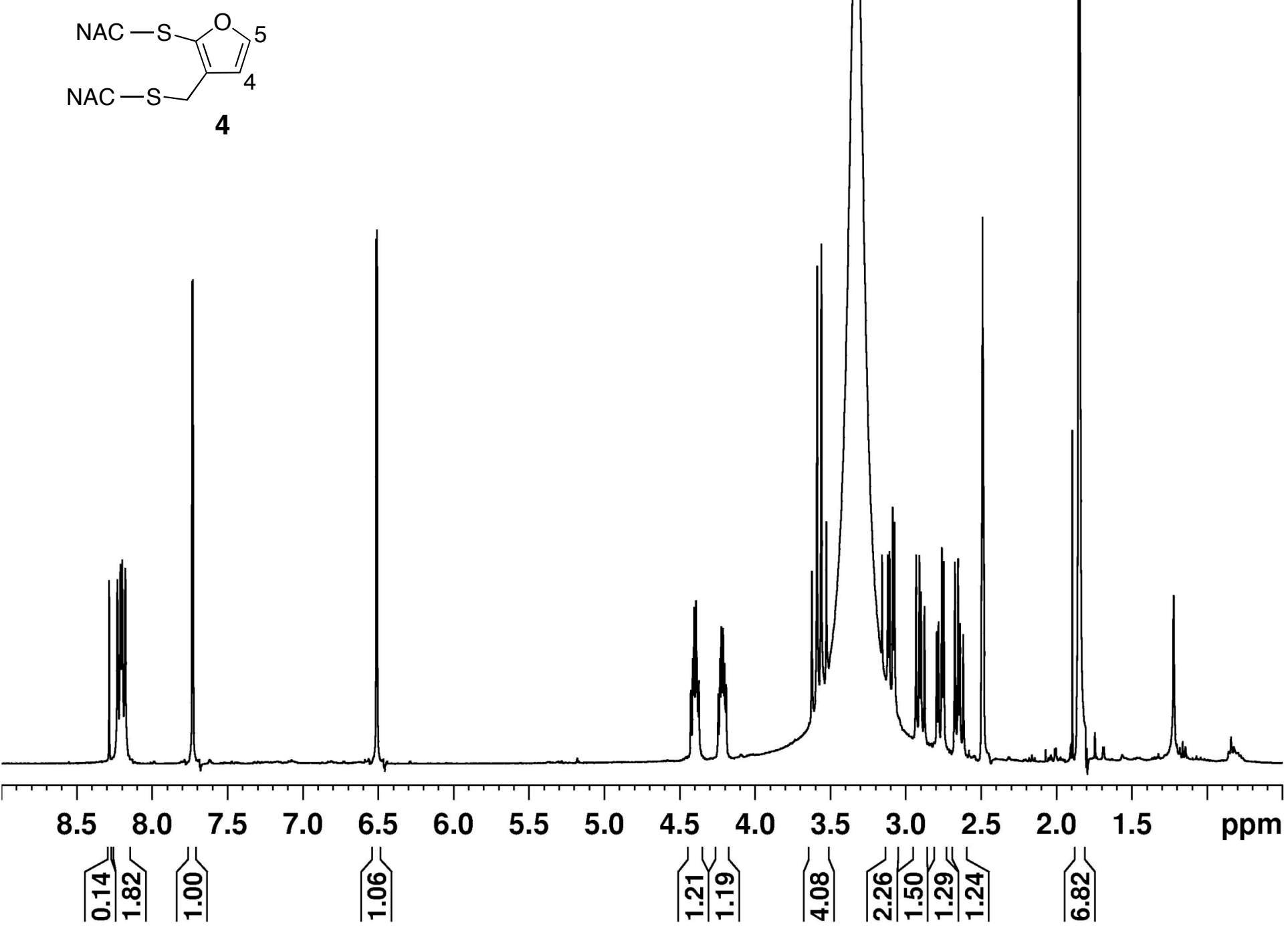
Suppl. Figure 6. COSY spectrum of $4(400 \mathrm{MHz})$.

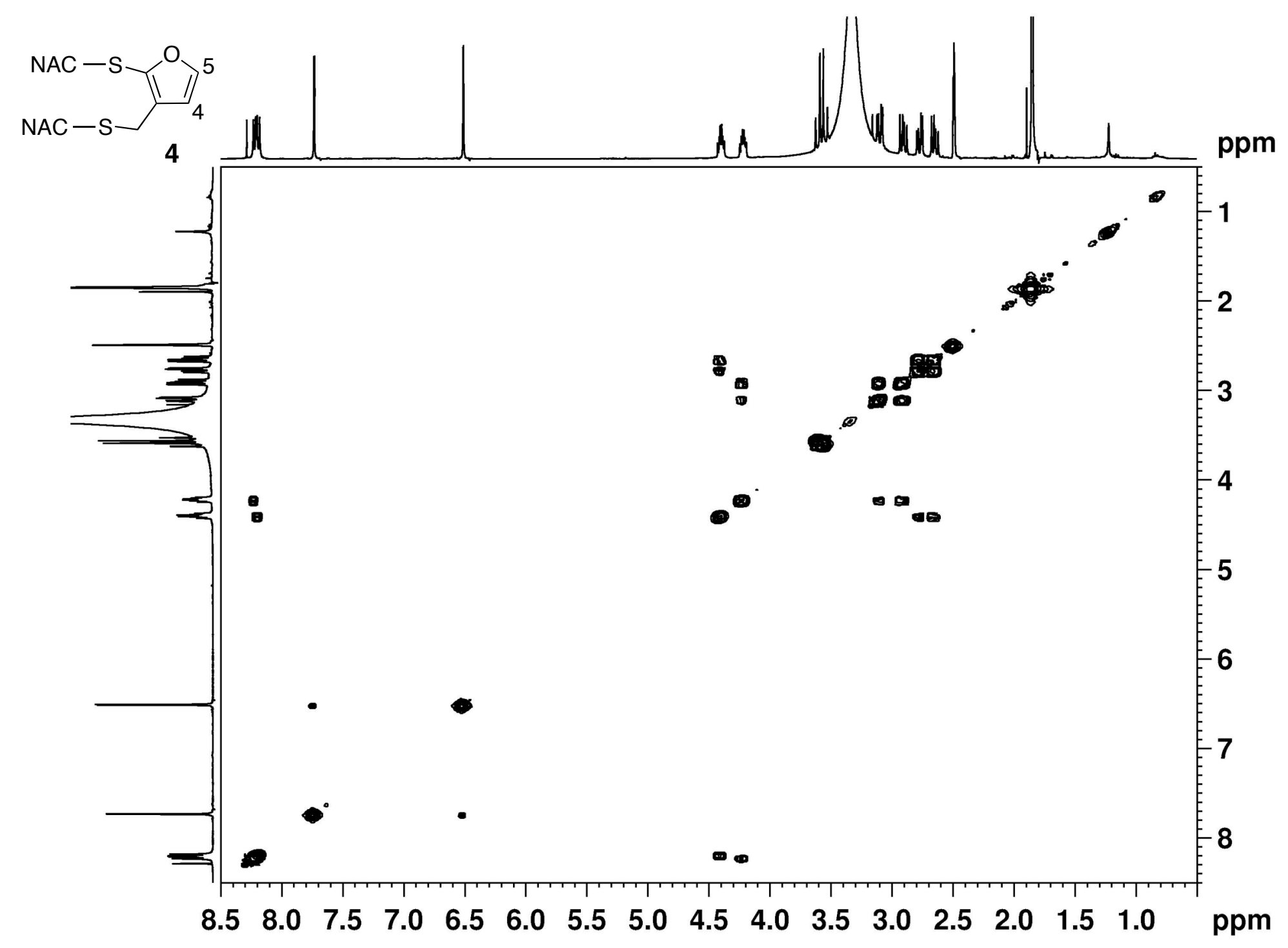


Suppl. Figure 7. ${ }^{1} \mathrm{H}$ NMR spectrum of $\mathbf{5 a}(400 \mathrm{MHz})$.

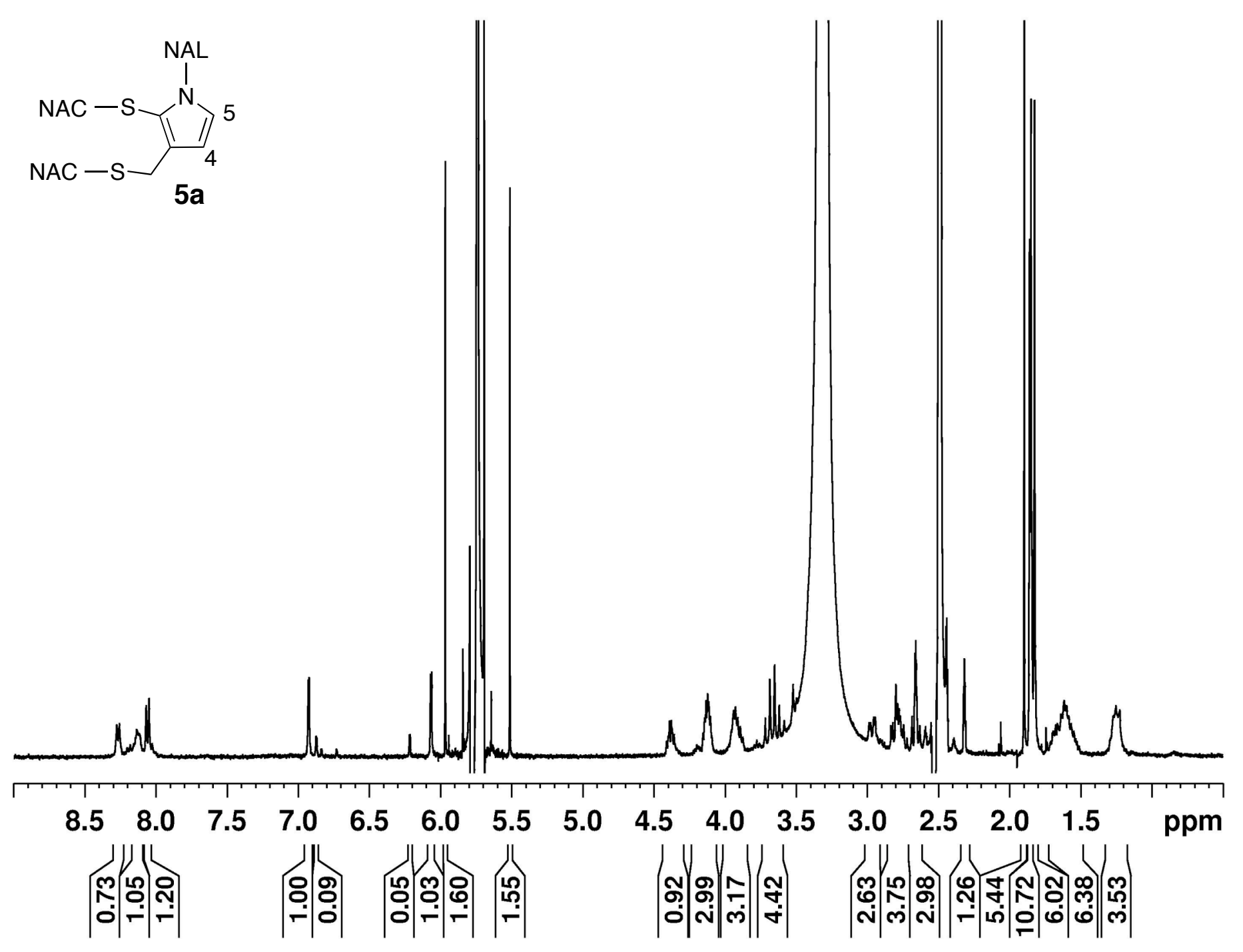


Suppl. Figure 8. COSY spectrum of $\mathbf{5 a}(400 \mathrm{MHz})$.

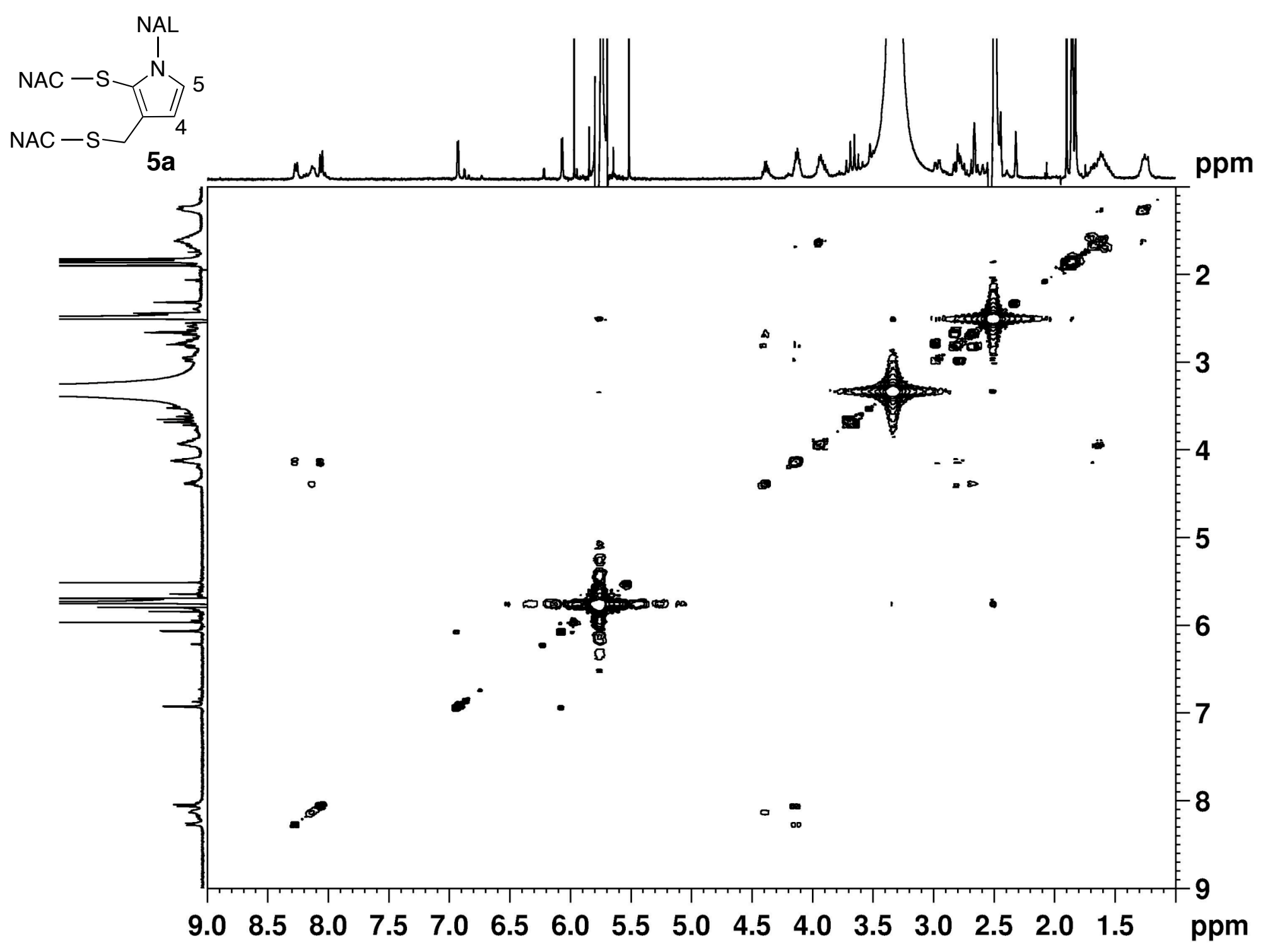


Suppl. Figure 9. ${ }^{1} \mathrm{H}$ NMR spectrum of $\mathbf{5 b}(400 \mathrm{MHz})$.
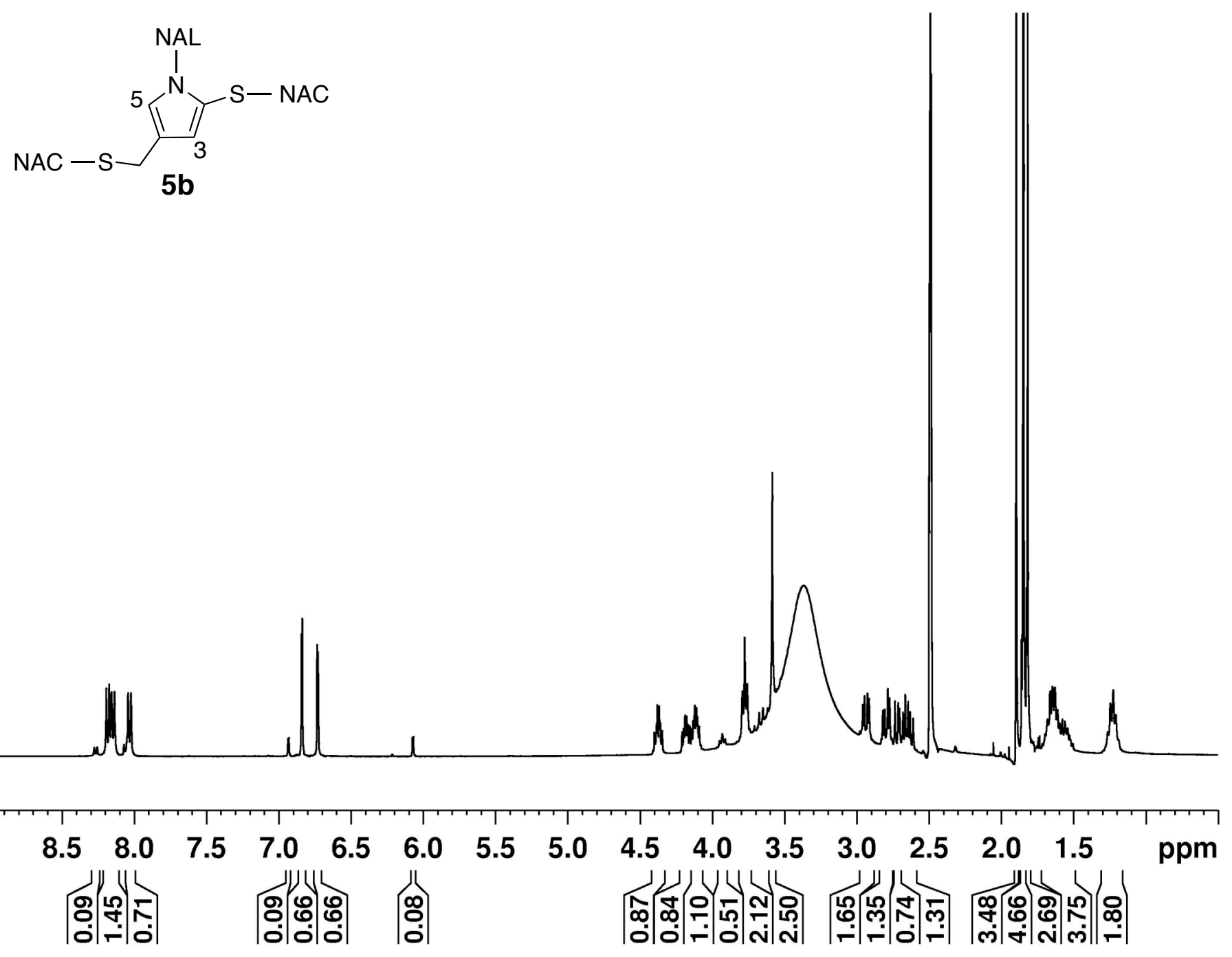
Suppl. Figure 10. COSY spectrum of $\mathbf{5 b}(400 \mathrm{MHz})$.

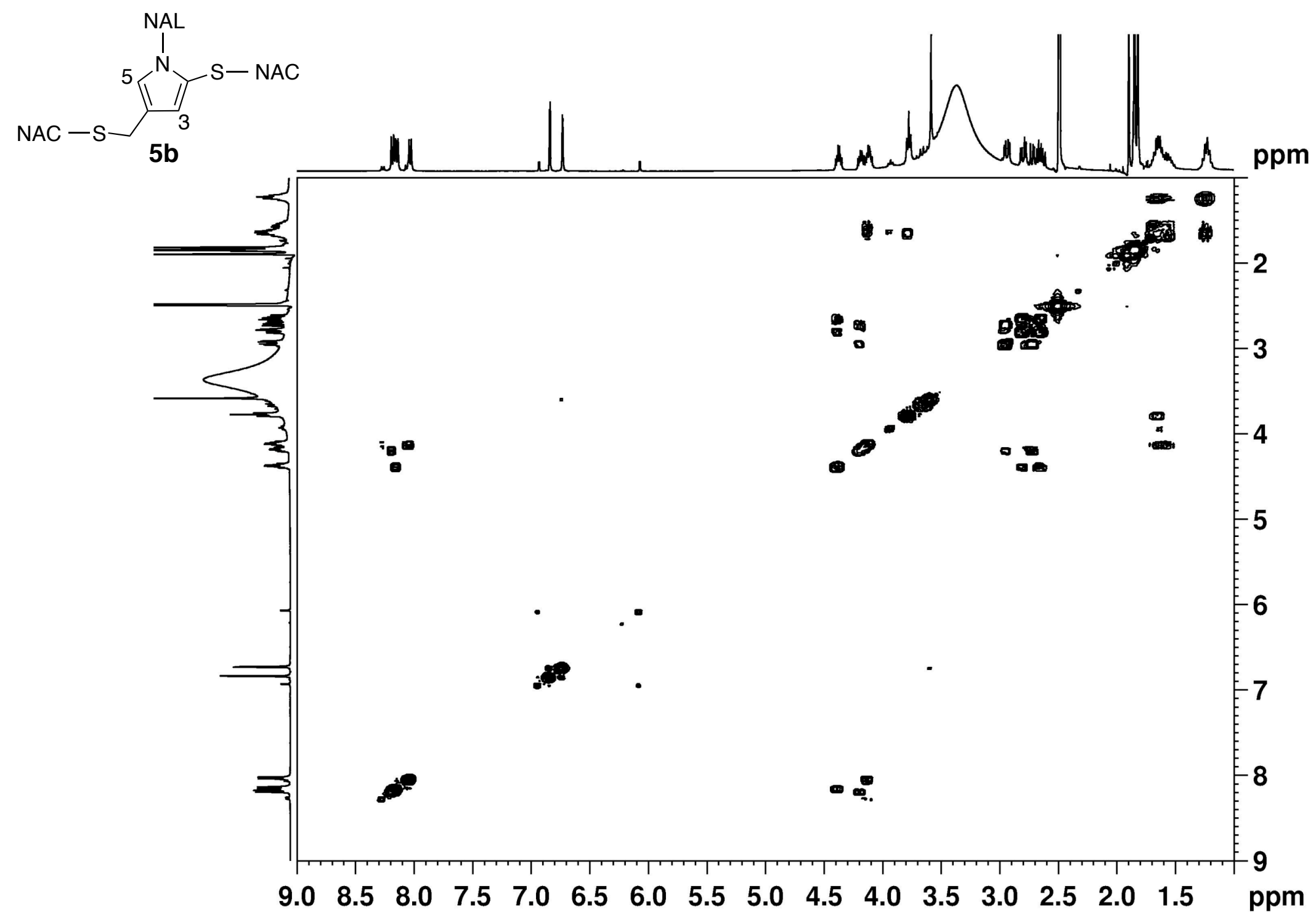




\section{Suppl. Figure 11. ${ }^{1} \mathrm{H}$ NMR spectrum of $\mathbf{7 c}, \mathbf{d}(400 \mathrm{MHz})$.}

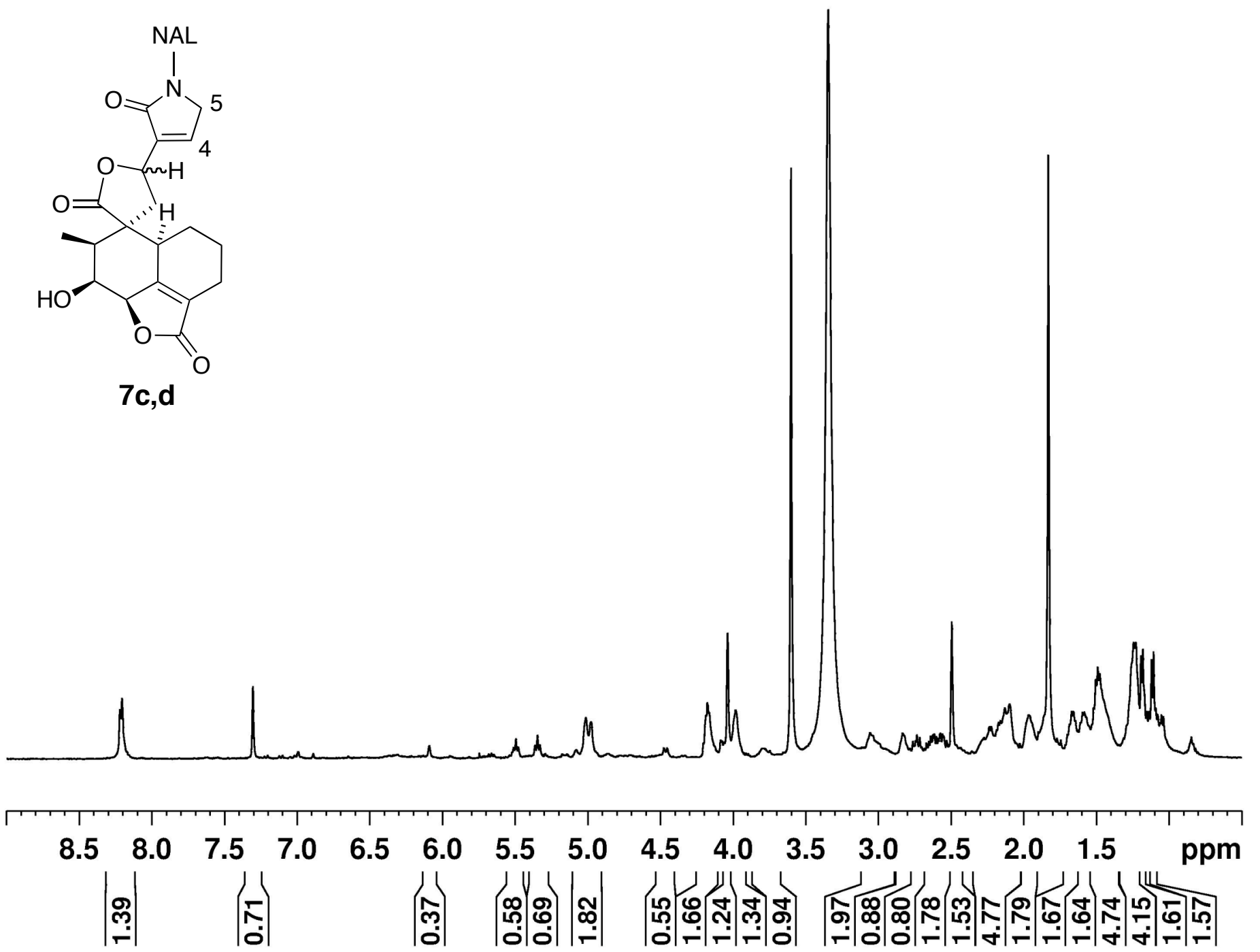


Suppl. Figure 12. COSY spectrum of $7 \mathbf{c}, \mathbf{d}(400 \mathrm{MHz})$.

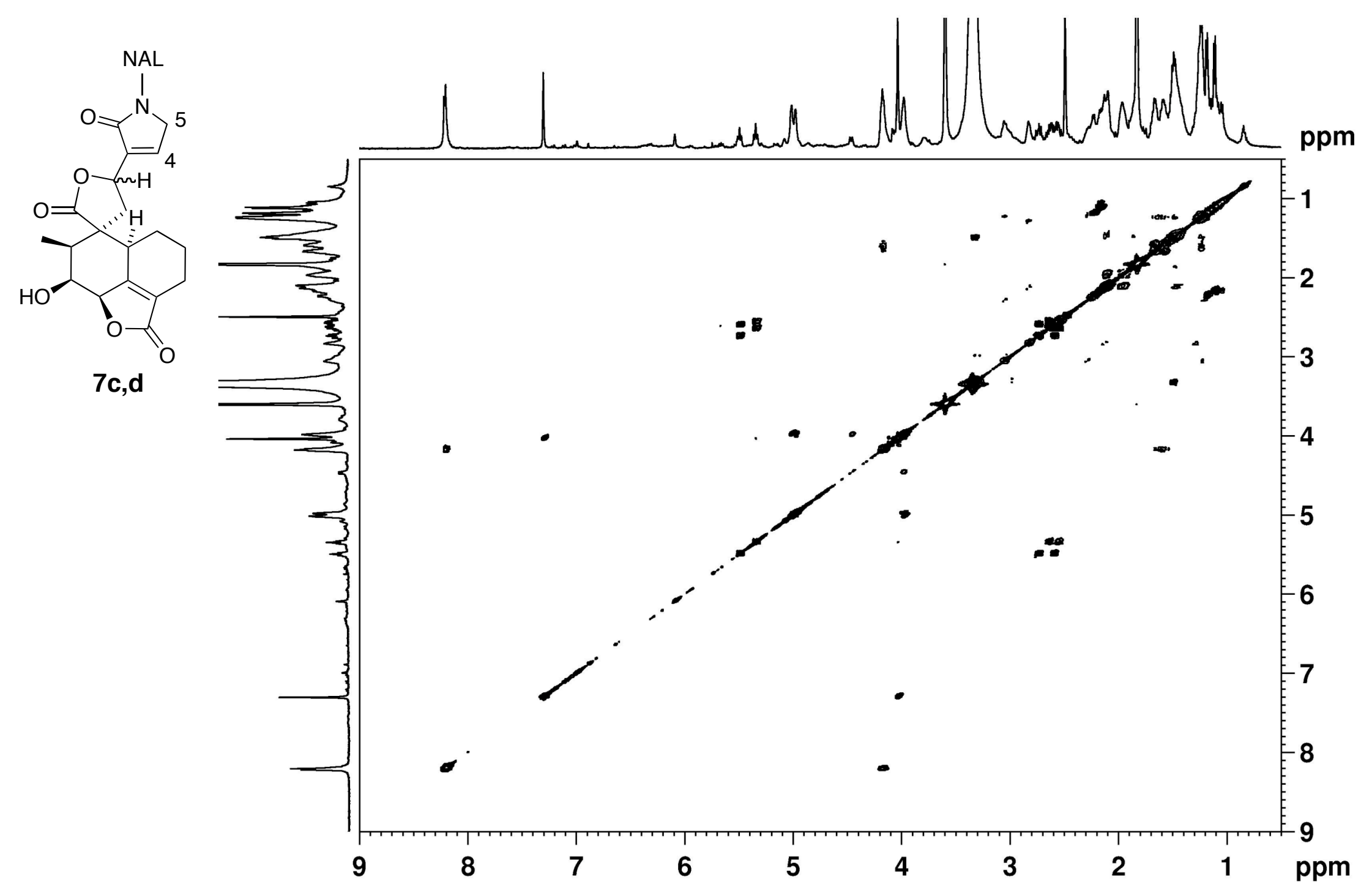


Suppl. Figure 13. ${ }^{1} \mathrm{H}$ NMR spectrum of $8 \mathrm{c}(400 \mathrm{MHz})$.

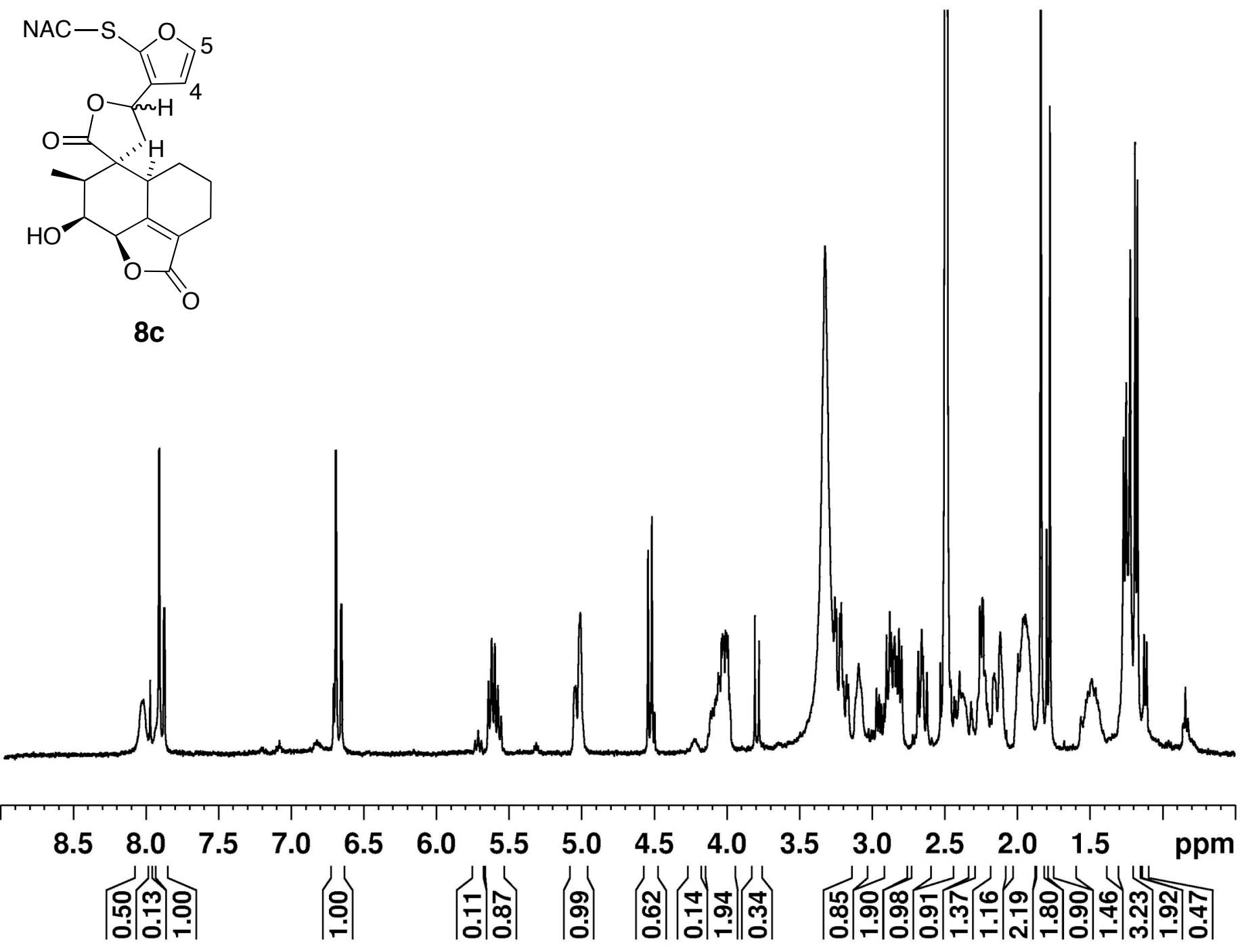


Suppl. Figure 14. COSY spectrum of $8 \mathbf{c}(400 \mathrm{MHz})$.

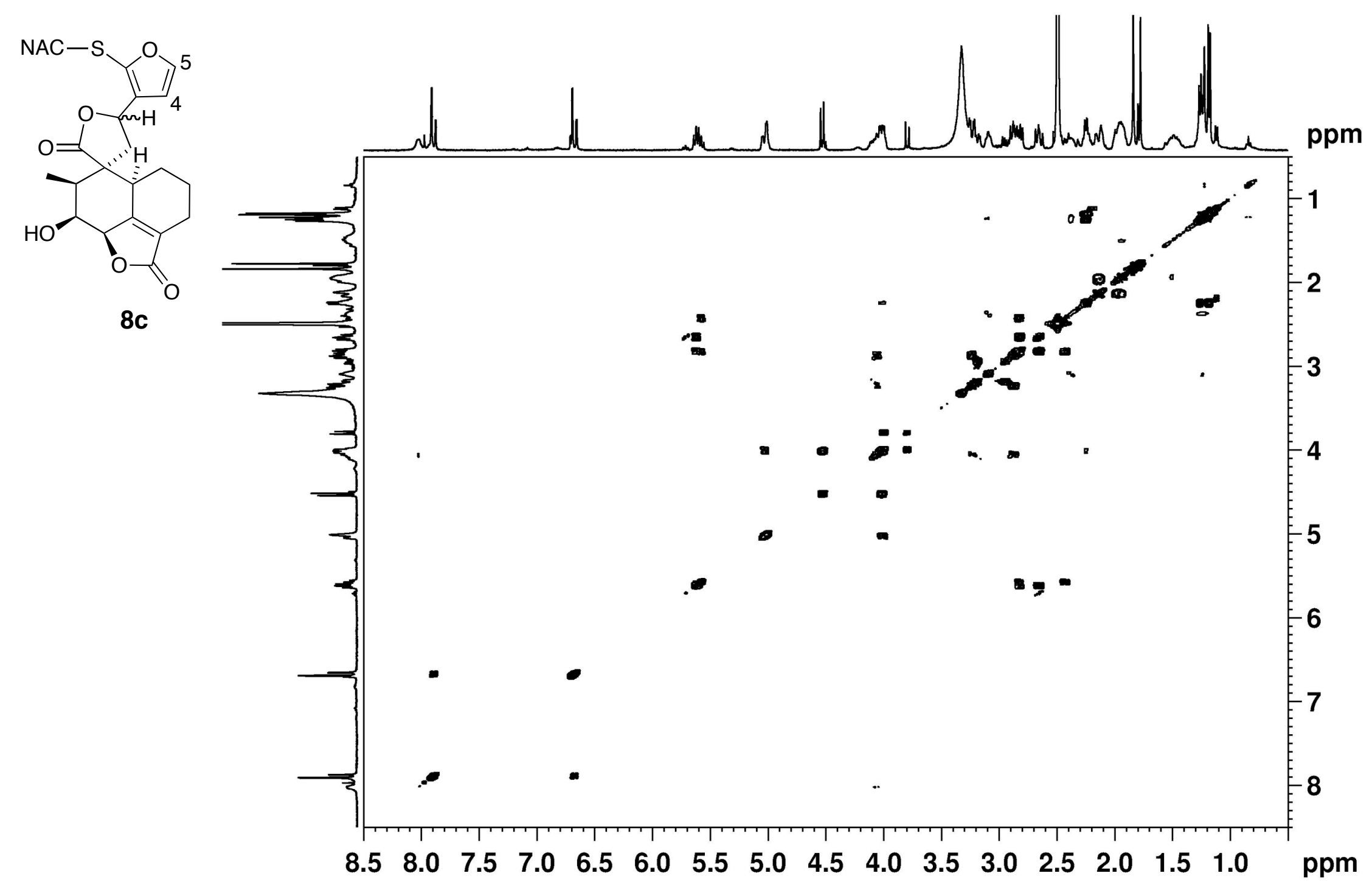


Suppl. Figure 15. ${ }^{1} \mathrm{H}$ NMR spectrum of $9 \mathrm{c}(400 \mathrm{MHz})$.

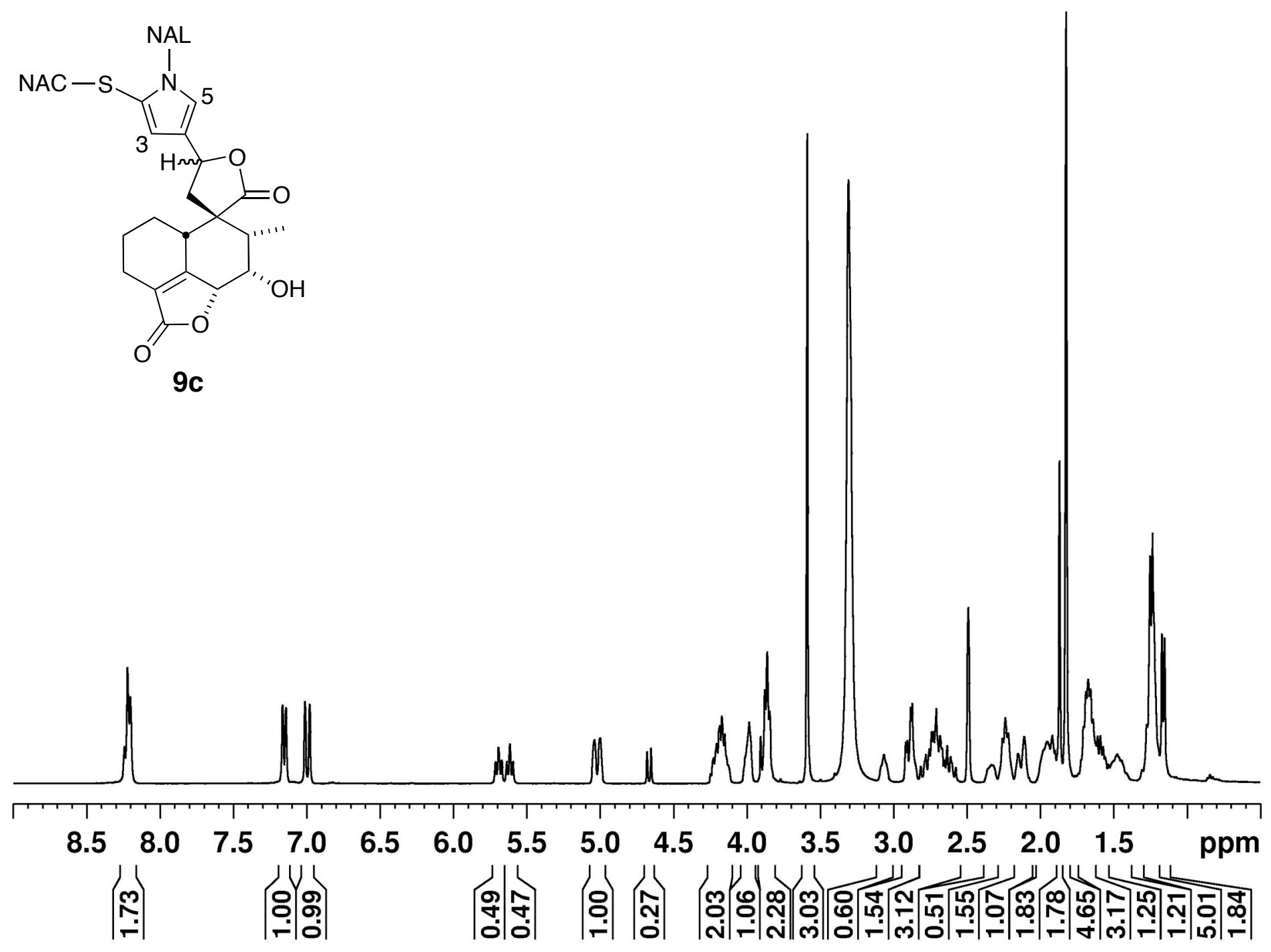


Suppl. Figure 16. COSY spectrum of $9 \mathrm{c}(400 \mathrm{MHz})$.

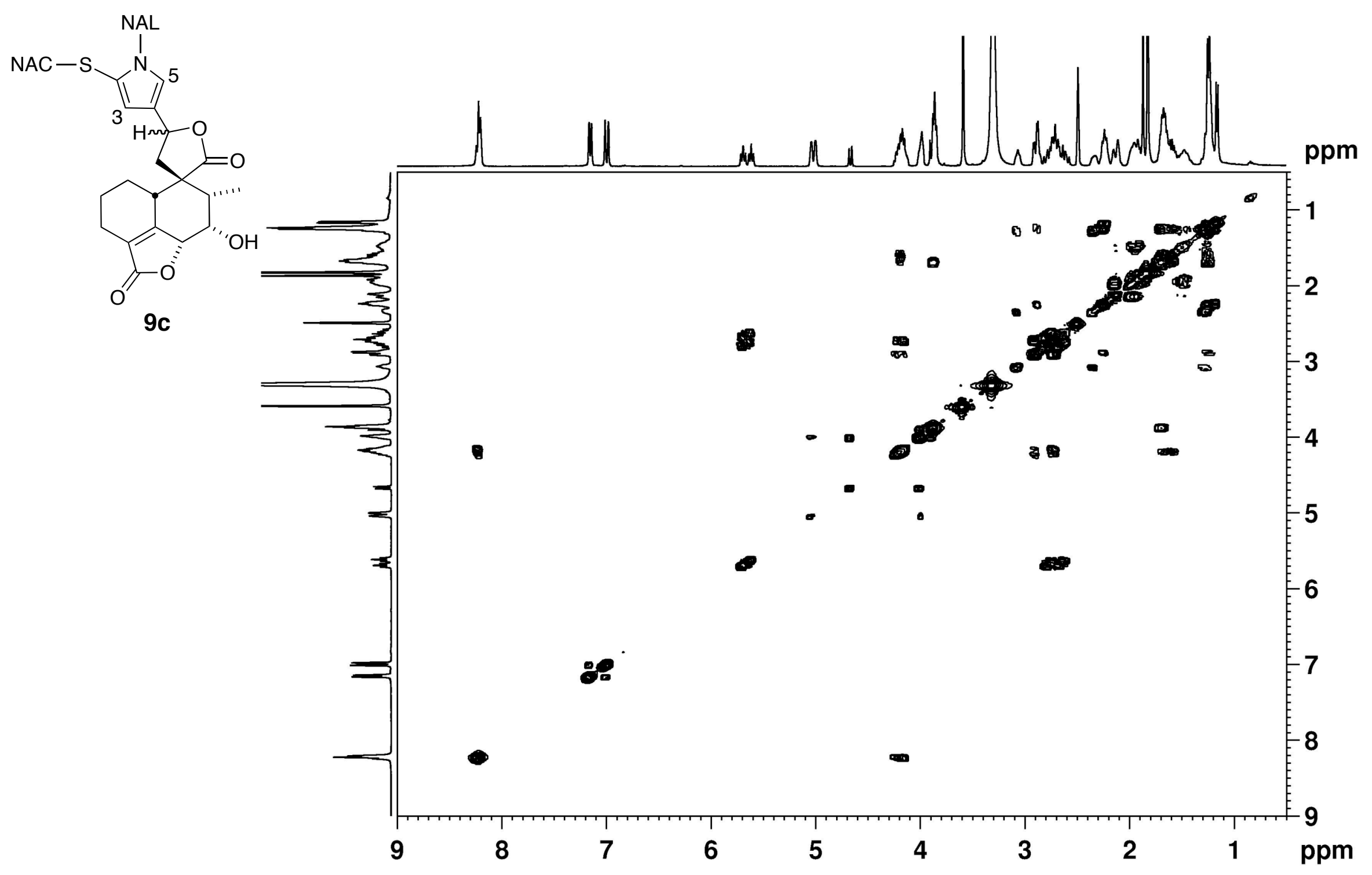

